\title{
Effiziente und effektive Gassensorkalibrierung mit randomisierten Gasmischungen
}

\author{
Tobias Baur, Manuel Bastuck, Caroline Schultealbert, Andreas Schütze und Tilman Sauerwald
}

\section{Lehrstuhl für Messtechnik, Universität des Saarlandes, Saarbrücken, Deutschland} Kontakt: t.baur@Imt.uni-saarland.de.de

\section{Einleitung}

Die selektive Quantifizierung von Zielgasen in komplexen Mischungen ist ein integraler Bestandteil zahlreicher Zielanwendungen chemischer Gassensoren, wie zum Beispiel Raumluftüberwachung und Brandfrüherkennung. Die Daten der Sensoren werden dafür üblicherweise mit statistischen Modellen ausgewertet, die wiederum im Labor kalibriert wurden.

In den meisten wissenschaftlichen Veröffentlichungen wird die Kalibrierung lediglich als ein Mittel zum Zweck betrachtet, um die Performance eines neuen Materials oder einer neuen Datenverarbeitungsmethode zu zeigen. Das experimentelle Design besteht meist aus einigen wenigen, festen Konzentrationsstufen pro Gas und der Sensor wird stets nur einem Zielgas gleichzeitig ausgesetzt [1, 2]. Die resultierenden Daten sind relativ einfach in Bezug auf Empfindlichkeit, Selektivität und Reaktionsgeschwindigkeit zu bewerten, vernachlässigen aber die Komplexität im Feld. Die Sensorkalibrierung mit sequenziellen Einzelgasangeboten bzw. Variation weniger Konzentrationsstufen [3, 4] kann zur Vernachlässigung von Maskierungseffekten bzw. Gaswechselwirkungen sowie zu Überanpassung durch systematische Einflüsse führen.

In diesem Beitrag präsentieren wir eine auf randomisierten Gasmischungen beruhende Kalibriermethode [5]. Dabei wird in jedem Zeitfenster für jedes beteiligte Gas eine zufällige Konzentration aus einer vorgegebenen Verteilung gezogen. So kann schnell eine nahezu beliebig große Anzahl einzigartiger Gasgemische erzeugt werden, die jeweils alle Kalibriergase enthalten. Der Ansatz ist einfach zu konfigurieren und anzuwenden, kann einfach für eine Vielzahl von Zielanwendungen angepasst werden und ist nachweislich der sequenziellen Kalibrierung an Effizienz und Effektivität deutlich überlegen [6].

\section{Messaufbau}

In dem hier vorgestellten Messaufbau wurden insgesamt elf unterschiedliche Sensoren gemessen, davon sieben Halbleitergassensoren (MOS) und vier gassensitive Feldeffekttransistoren (FET): AS-MLV, AS-MLV-P2, ein mit Siloxan für Wasserstoffselektivität konditionierter AS-MLV-P2 (ams sensor solutions Germany), UST GGS1xxx (Prototyp, Umweltsensortechnik), SGP30 (Sensirion), BME680 (Bosch Sen- sortec), ZMOD4410 (IDT) und vier unterschiedliche FET-Prototypen (SenSiC AB, Kista, Schweden). Alle Sensoren wurden nicht in den Betriebsmodi betrieben, die von den jeweiligen Herstellern empfohlen werden, sondern in einem selbst designten temperaturzyklischen Betrieb (Temperature Cycled Operation, TCO) $[7,8]$. Das Design des TCO für die MOSSensoren beruht auf dem DSR-Verfahren (Differential Surface Reduction) [9].

Die MOS-Sensoren ohne integrierte Elektronik (Sensoren von ams sensor solutions und UST) wurden mit der am Lehrstuhl für Messtechnik entwickelten Elektronik mit logarithmischer Leitwertmessung und widerstandsbasierter Temperaturreglung betrieben [10]. Die Temperatureinstellung/-regelung und Leitwertauslesung der digitalen MOS-Sensoren (SGP30, BME680, ZMOD4410) wurden über die integrierten ASICs vorgenommen und das Timing der Zyklen des TCO wurde von einem MikrokontrollerBoard gesteuert. Die Ergebnisse und Erkenntnisse dieses Manuskripts beruhen auf dem Sensor ASMLV-P2 (ohne Konditionierung), da hierzu die größte Erfahrung in der Anwendung des DSR-Verfahrens besteht $[10,11,12]$. Der Temperaturzyklus des ASMLV-P2 mit einer Länge von $120 \mathrm{~s}$ ist in Abb. 1 dargestellt.

Die Gasgemische werden in einer Gasmischanlage (GMA) $[13,14]$ erzeugt. Die GMA besteht aus mehreren Massenflussreglern (MFC), um Trägergas (Nullluft) und definierte Gaskonzentrationen aus Gasflaschen bereitzustellen. Für die Erzeugung sehr niedriger reproduzierbarer Gaskonzentrationen und um einen größeren Dynamikumfang zu erreichen, verfügt die GMA über Gaslinien mit zweifacher Verdünnung. Die Feuchte wird durch einen einstellbaren Trägergasfluss generiert, welcher durch eine Waschflasche mit HPLC-Wasser bei einer Temperatur von $20^{\circ} \mathrm{C}$ geleitet wird.

Um die Beeinflussung der Sensoren untereinander gering zu halten, wurde der Gesamtfluss von $400 \mathrm{ml} / \mathrm{min}$ in vier gleich große Flüsse aufgeteilt. Für die Aufteilung wurde vor jeder Linie eine Restriktion $\left(10 \mathrm{~cm}, 1 / 16^{\prime \prime}\right.$ Edelstahlrohr) installiert, die im Vergleich zum restlichen Aufbau ( $<25 \mathrm{~cm}$, min. 1/8“) den Flusswiderstand dominiert. Die Sensorkammern sind aus PTFE und Aluminium gefertigt. 


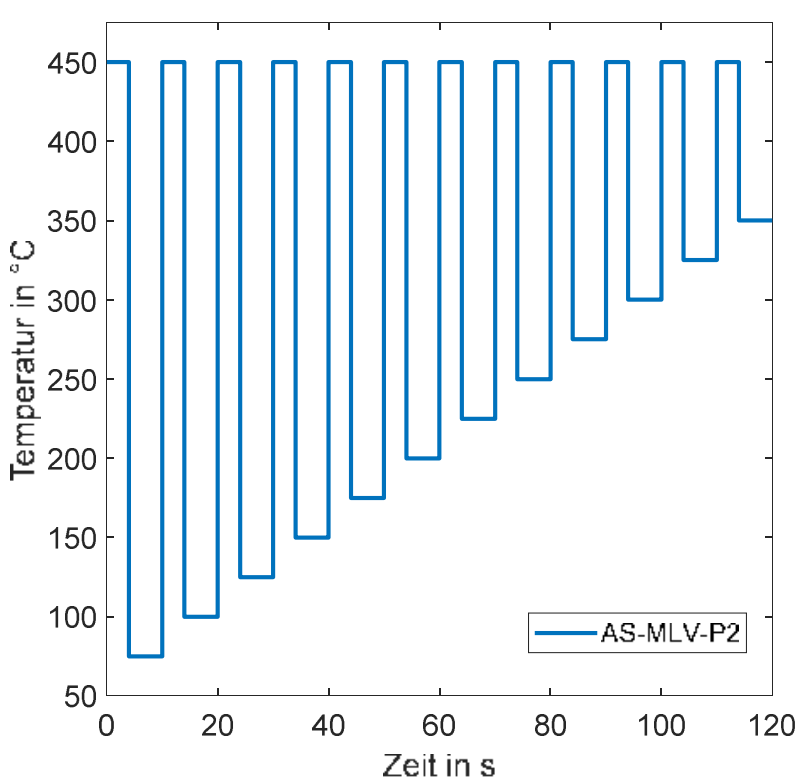

Abb. 1: Temperaturzyklus des AS-MLV-P2.

\section{Kalibriermethode}

Die Kalibriermethode mit randomisierten Gasgemischen wird am Beispiel der Bewertung der Raumluftqualität gezeigt, kann jedoch auf beliebige Anwendungen und Zielgrößen angewendet werden. Die relevanten Gase für die Bewertung der Raumluftqualität können in zwei Gruppen, Hintergrund (Nullluft, Feuchte und anorganische Störgrößen) und (sehr) flüchtige organische Verbindungen (V)VOC, eingeteilt werden. Eine schematische Darstellung der Generierung randomisierter Gasgemische ist in Abb. 2 dargestellt.

Das Ziel der Bewertung könnte beispielsweise die Quantifizierung von TVOC (total VOC) sein, um die Klassifikation des TVOC-Levels nach dem Umweltbundesamt [15] vorzunehmen. Alternativ können die (V)VOC in Hintergrund- und Zielgrößen unterteilt werden mit dem Ziel, die Menge an toxischen oder karzinogenen Substanzen (hier Benzol und Formaldehyd) zu quantifizieren. Das Umweltbundesamt führt in [16] die statistische Verteilung von über 300 verschiedene (V)VOC in Innenräumen auf. Alle diese (V)VOC in variablen Verhältnissen zu vermessen ist anlagentechnisch nicht umsetzbar, so dass eine Vorauswahl auf Basis der zu erwartenden Konzentration zu treffen ist. Da wir mit dem verwendeten Aufbau apparativ auf sechs Gase beschränkt sind wurden je zwei Repräsentanten für anorganische Hintergrundgase, Hintergrund- und Ziel-VOC gewählt. Für den anorganischen Hintergrund wurden die Gase Wasserstoff $\left(\mathrm{H}_{2}\right)$ und Kohlenstoffmonoxid (CO) gewählt. Der (V)VOC Hintergrund wurde durch die Gase Aceton und Toluol repräsentiert. Die karzinogenen Stoffe Formaldehyd und Benzol wurden als Ziel-VOC gewählt.

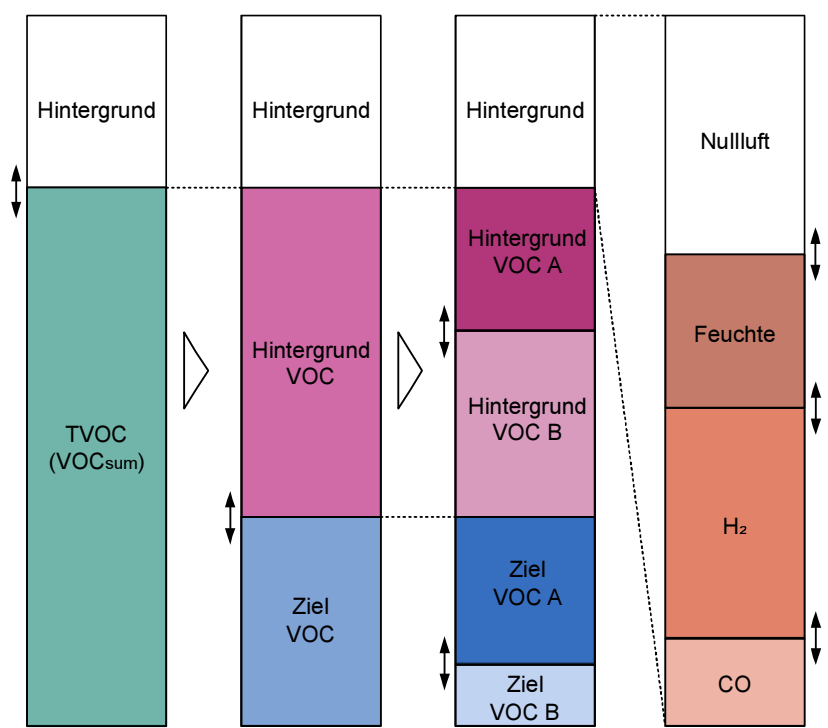

Abb. 2: Schematische Darstellung der randomisierten Generierung von Gasgemischen (modifiziert nach [6]).

$\mathrm{Da}$ in dieser Konfiguration lediglich flüchtige organische Gase betrachtet werden, wird die Summe aller gemessenen (V)VOC als VOC $_{\text {sum }}$ definiert, um dies klar vom gebräuchlichen TVOC-Begriff abzugrenzen. Die randomisierten Gasgemische wurden mit einem Python-Skript [6] generiert. Zur Generierung eines randomisierten Gasgemisches wurde die Zusammensetzung der Hintergrund-Komponenten $\left(\mathrm{CO}, \mathrm{H}_{2}\right.$, Feuchte) und $\mathrm{VOC}_{\text {sum }}$ unabhängig voneinander variiert. Die Konzentrationen von Feuchte, $\mathrm{CO}, \mathrm{H}_{2}$ und $\mathrm{VOC}_{\text {sum }}$ sind jeweils gleichverteilt. Das Verhältnis aus Hintergrund- und Ziel-VOC in VOC $_{\text {sum }}$ wird in einem ersten Schritt zufällig gewählt (zwischen 0 und $20 \%$ Ziel-VOC). Die Aufteilung von Ziel- und Hintergrund-VOC in einzelne Gase erfolgt auf die gleiche Art und Weise. Die Parameter für die Generierung sind in Tab. 1 dargestellt. Die Intervallgrenzen des anorganischen Hintergrunds $\mathrm{H}_{2}$ und $\mathrm{CO}$ sind der atmosphärische Hintergrund [17] und hohe, tatsächlich gemessene Werte in der Innenraumlauft [15, 16]. Dieselben Messungen liegen auch der Verteilung von $\mathrm{VOC}_{\text {sum }}$ zugrunde.

Tab. 1: Parameter der randomisierten Messungen.

\begin{tabular}{|l|c|}
\hline & Konzentrationsbereich \\
\hline Wasserstoff & $500-2500 \mathrm{ppb}$ \\
\hline Kohlenstoffmonoxid & $100-2000 \mathrm{ppb}$ \\
\hline Feuchte & $25-75 \% \mathrm{RH}$ \\
\hline VOC $_{\text {sum }}$ & $100-5000 \mu \mathrm{g} / \mathrm{m}^{3}$ \\
\hline Anteil der Ziel-VOC & $0-20 \%$ \\
\hline
\end{tabular}

Durch einen Fehler im Messaufbau, wurde Toluol als Ziel-VOC angeboten und Benzol als HintergrundVOC. Daher sind die angebotenen Konzentrationen des karzinogenen Benzols in dieser Studie unverhältnismäßig hoch, während Toluol sich lediglich im 
niedrigen ppb-Bereich bewegt. Auf die allgemeinen Schlussfolgerungen über die Effizienz des Kalibrierverfahrens hat dies keine Auswirkungen, die Ergebnisse zur selektiven Quantifizierung dieser beiden VOCs sollten jedoch entsprechend vorsichtig interpretiert werden. Die Verteilungen der einzelnen Gase sind in [6] zu finden. Jedes randomisierte Gasgemisch wurde den Sensoren je 20 min lang angeboten. Insgesamt wurden 800 unterschiedliche Gasgemische getestet.

Um einen Vergleich zu einer gängigen sequenziellen Kalibrierstrategie (ein Gas nach dem anderen, aufsteigende Konzentrationswerte) zu ermöglichen, wurde im ersten Schritt ein solches Gasprofil gemessen. Jedes Gas wurde in vier verschiedenen Konzentrationen für jeweils 20 min angeboten (vgl. Tab. 2). $\mathrm{CO}$ und $\mathrm{H}_{2}$ wurden, außer, wenn sie Zielgas waren, während der Messung durchgehend auf ihrer atmosphärischen Konzentration von $500 \mathrm{ppb}_{2}$ und 150 ppb CO gehalten. Das sequenzielle Profil wurde dreimal mit unterschiedlichen Feuchten (25, 50 und $75 \% \mathrm{RH}$ ) wiederholt. Insgesamt besteht das sequenzielle Profil aus 72 verschiedenen Gasangeboten.

Tab. 2: Gaskonzentrationen der sequenziellen Messungen

\begin{tabular}{|l|c|c|c|c|}
\hline Gas & \multicolumn{4}{|c|}{ Konzentration in ppb } \\
\hline Aceton & 250 & 500 & 750 & 1000 \\
\hline Benzol & 250 & 500 & 750 & 1000 \\
\hline Kohlenstoffmonoxid & 150 & 300 & 450 & 600 \\
\hline Formaldehyd & 40 & 80 & 120 & 160 \\
\hline Wasserstoff & 500 & 750 & 1000 & 1250 \\
\hline Toluol & 5 & 25 & 45 & 65 \\
\hline
\end{tabular}

\section{Auswertung}

Die Auswertung wurde mit der Open-SourceSoftware $D A V^{3} E$ [18] durchgeführt und kann in die Schritte Merkmalsextraktion, Dimensionsreduktion, Regression, Validierung und Testen unterteilt werden.

$\mathrm{Zu}$ Beginn wird aus den acht Sensorzyklen jedes Gasangebots durch Mittelwertbildung ein Zyklus erzeugt. Für die Merkmalsextraktion wird der $120 \mathrm{~s}$ lange Zyklus in 120 äquidistante Bereiche unterteilt. In diesen Bereichen werden jeweils Mittelwert und Steigung berechnet. D.h. für jeden Sensor werden pro Zyklus insgesamt 240 Merkmale extrahiert.

Zur Verhinderung einer Überanpassung bei der Modellbildung wird eine Dimensionsreduktion nach der Merkmalsextraktion durchgeführt. Dazu werden von jedem Sensor die besten 20 Merkmale mittels einer Hauptkomponentenanalyse (PCA) selektiert.

Die Quantifizierung der gewünschten Zielgröße (Einzelgas oder Gasgemisch, bspw. VOC $_{\text {sum }}$ ) wird mit einer Regression (PLSR, partial least squares regression) durchgeführt. Für die Validierung und das Testen wurde das 6-fache-Kreuzvalidierungsverfahren gewählt.

Für den Vergleich der Fähigkeit für jedes einzelne Gases im untersuchten Konzentrationsbereich bei Kompensation des Hintergrunds zu quantifizieren, wird ein Performancewert

$$
\text { performance }=\operatorname{std}(c(g)) / \operatorname{RMSEP}(g)-1
$$

definiert [6]. Dabei ist $\operatorname{std}(\mathrm{c}(\mathrm{g}))$ die Standardabweichung der eingestellten Konzentrationen für ein Gas $\mathrm{g}$ und $\operatorname{RMSEP}(\mathrm{g})$ das mittlere Abweichungsquadrat (RMSE) der Vorhersage eines PLSR-Modells für die Quantifizierung eines Testdatensatzes dieses Gases. Mit der Performance lässt sich abschätzen, wie gut ein Sensor ein Gas im gegebenen Konzentrationsbereich dieser Anwendung Quantifizieren kann. Es ist jedoch kein allgemein gültiger Performancewert für die Quantifizierung eines Gases, das in andere Anwendungen einfach übertragen werden kann.

\section{Ergebnisse}

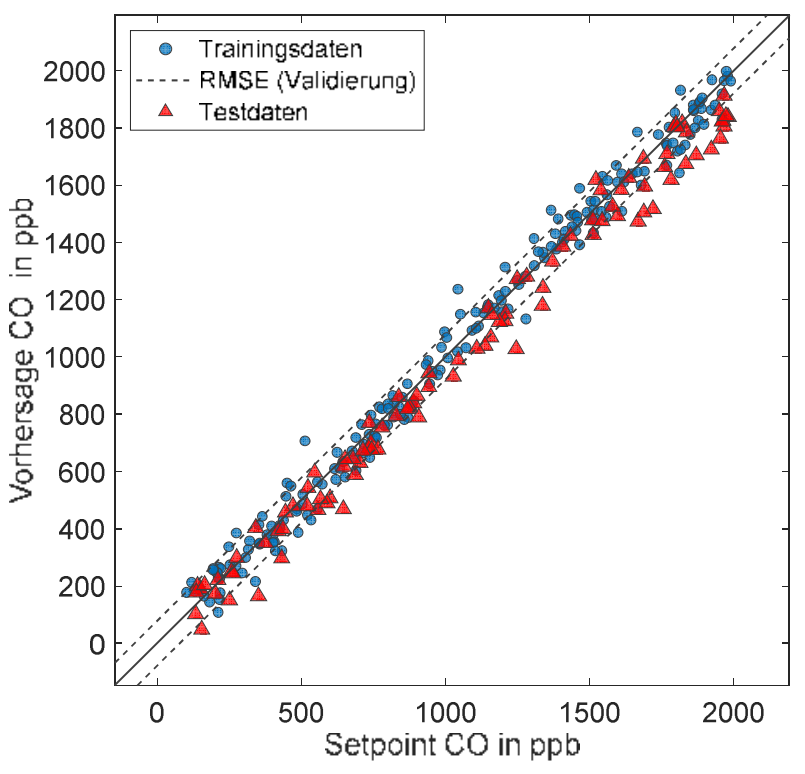

Abb. 3: PLSR-Modell für den AS-MLV-P2 für die Quantifizierung von CO. Berechnung des Modells aus einem reduzierten Datensatz mit 300 randomisierten Gasgemischen; Training, Validierung und Testen mit 100 Gasangeboten.

Abb. 3 zeigt beispielhaft ein PLSR-Modell für den AS-MLV-P2 zur Quantifizierung des Gases CO, das mit 100 randomisierten Gasangeboten (blau) trainiert wurde. Der in der Validierung mit 100 weiteren Gasangeboten ermittelte RMSE ist als Maß für die Varianz des Modells durch gestrichelte Linien dargestellt. Die roten Punkte zeigen die vorhergesagten Punkte aus 100 weiteren Gasangeboten, die weder im Training noch in der Validierung verwendet wur- 
den. Dabei ist eine gute Übereinstimmung des Testdatensatzes mit den Trainingsdaten zu erkennen.

Für die Berechnung der Performance der einzelnen Regressionsmodelle werden die Modelle aus einem Datensatz mit 400 randomisierten Gasgemischen mit 6-facher Kreuzvalidierung trainiert, validiert und getestet. Die einzelnen Performancewerte der $\mathrm{Re}-$ gressions-Modelle des AS-MLV-P2 für die verschiedenen Zielgrößen sind in Abb. 4 dargestellt. CO zeigt die höchste Performance aller getesteten Einzelgase, d.h. der AS-MLV-P2 kann CO vor einem variablen Hintergrund aller anderen Gase gut quantifizieren. Auch für Aceton, Wasserstoff und Wasser zeigen die Modelle gute Ergebnisse. Die Performance für Benzol ist mit einem Wert von 2 in Anbetracht der hohen Konzentrationen relativ gering und auch Toluol und Formaldehyd können in dem komplexen Hintergrund nicht selektiv quantifiziert werden. Die Quantifizierung von $\mathrm{VOC}_{\text {sum }}$ wiederum funktioniert unabhängig von der Einheit $\left(\mu \mathrm{g} / \mathrm{m}^{3}\right.$ oder $\mathrm{ppb})$.

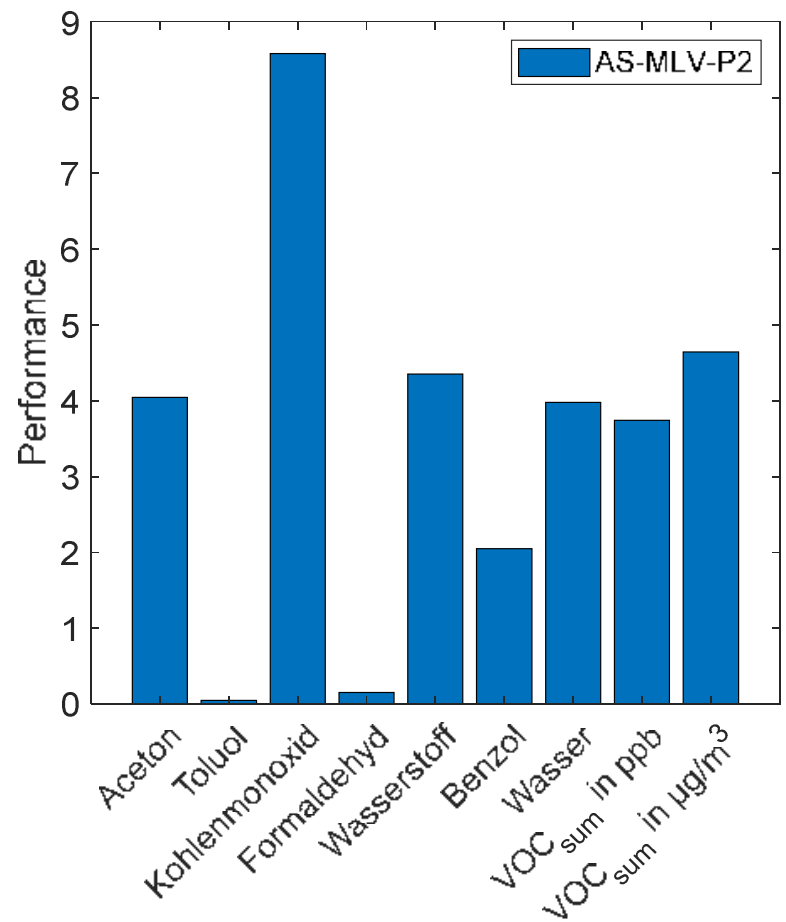

Abb. 4: Performance des AS-MLV-P2 bei der Quantifizierung unterschiedlicher Zielgrößen; Datensatz mit 400 randomisierten Gasgemischen; Validierung und Testen mit 6-facher Kreuzvalidierung.

Für einen Vergleich zwischen randomisierter und sequenzieller Kalibiermethode werden drei Kombinationen untersucht: (1) Training/Validierung randomisiert, Testen randomisiert; (2) Training/Validierung randomisiert, Testen sequenziell; und (3) Training/Validierung sequenziell, Testen randomisiert. Die Validierung erfolgt immer mit 6facher Kreuzvalidierung. Für einen fairen Vergleich werden alle Gasgemische mit einer Gaskonzentrati- on der Zielgröße, die nicht im Bereich der sequenziellen Kalibrierung enthalten ist, in der Berechnung ignoriert. $\mathrm{Da}$ die letzten sechs Gasangebote ( $75 \% \mathrm{RH}$, Benzol 750 und $100 \mathrm{ppb}$ und alle Formaldehyd-Angebote) im Datensatz der sequenziellen Messung fehlen, sind es insgesamt 66 sequenzielle Gasgemische. Diese 66 sequenziellen Gasangebote werden mit 66 randomisierten Gasgemischen im gleichen Konzentrationsbereich verglichen. Die Ergebnisse dieses Vergleichs sind in Abb. 5 dargestellt. Die beste Performance wird für randomisiertes Training mit randomisiertem Testen erzielt werden. Sequenzielles Training und randomisiertes Testen zeigt hingegen nur bei Kohlenmonoxid einen Performancewert deutlich größer null. Der umgekehrte Fall hingegen erreicht bei fünf von neun Zielgrößen eine Performance deutlich größer eins. Die randomisierten Daten sind offensichtlich herausfordernder in der Vorhersage, was durch den deutlich variableren Hintergrund zu erwarten ist. Dies ermöglicht gleichzeitig ein effizienteres Training näher an der Realität, da aus jedem Gasgemisch ein Datenpunkt für jedes Gas gewonnen wird.

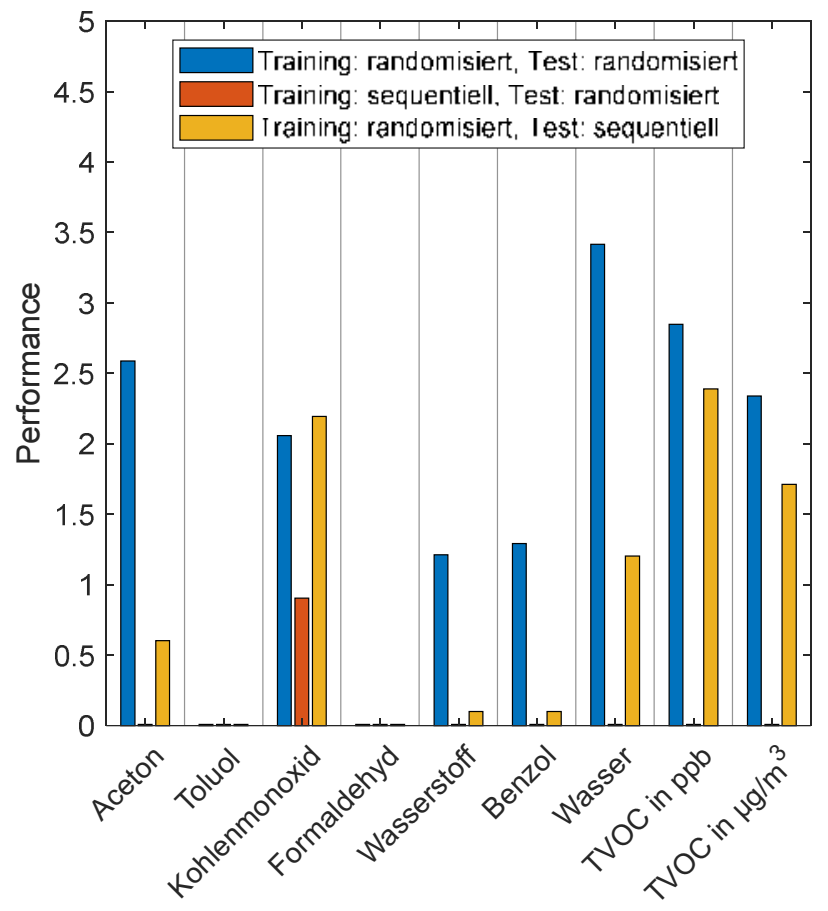

Abb. 5: Performance des AS-MLV-P2 bei der Quantifizierung unterschiedlicher Zielgrößen; Datensatz mit 132 Gasgemischen (132 randomisierte bzw. 66 randomisierte und 66 sequenzielle); Training und 6-fache Kreuzvalidierung mit 66 randomisierten (blau, gelb) bzw. 66 sequenziellen (rot) und Testen mit randomisierten Gasgemischen

(blau, rot) bzw. sequenziellen (gelb) Gasgemischen.

Der Vergleich der PLSR-Modelle in Abb. 6 und Abb. 7 zeigt, dass die klassische, sequenzielle Kalibrierung eine Untermenge der hier vorgestellten, 
randomisierten Kalibrierung ist. Das randomisiert trainierte Modell in Abb. 6 kann, trotz eines größeren RMSE, den sequenziellen Datensatz akkurat und präzise vorhersagen. Das sequenziell trainierte (und validierte) Modell in Abb. 7 erreicht einen besseren RMSE, scheitert jedoch an der Vorhersage des komplexeren, randomisierten Datensatzes. Die Messzeit zum Erzeugen beider Datensätze ist identisch.

\section{Zusammenfassung und Ausblick}

In diesem Beitrag wurde eine effiziente und effektive Gassensorkalibrierung mit randomisierten Gasmischungen vorgestellt. Die Ergebnisse wurden mit einer "klassischen“ Kalibrierung mit sequenziell ansteigenden Gaskonzentrationen verglichen. Während die sequenzielle Kalibrierung für keines der Gase ein Modell liefert, mit dem auch unbekannte Zusammensetzungen verschiedener Gase gute Vorhersagen getroffen werden können, kann die vorgestellte randomisierte Kalibrierung auch bei wenigen Testgasgemischen für eine ganze Reihe von Zielgasen zuverlässige Modelle erzeugen und ist damit deutlich effektiver. Für die Kalibrierung von Gemischen ist sie außerdem deutlich effizienter als kombinatorische Ansätze. Für den erfolgreichen Übergang von chemischen Sensoren aus dem Labor ins Feld bietet die vorgestellte Methode damit einen vielversprechenden Ansatz.

Da in der hier vorgestellten Untersuchung lediglich Einzelgase sequenziell vermessen wurden, sollte eine Untersuchung der Performance und Stabilität sequenzieller Kalibrierungen mit vollständig abgetasteten Kombinationen folgen im Vergleich zum randomisierten Ansatz. Ziel dieser Untersuchungen sollte sein, die ideale Anzahl randomisierter Gemische für den Erhalt eines zuverlässigen Modells zur Vorhersage der Konzentration eines Gases oder Gasgemisches zu bestimmen.

Die Konzentration der einzelnen Gase in der vorgestellten Messreihe sind gleichverteilt für die Hintergrundgase und $\mathrm{VOC}_{\text {sum. }}$. Durch die Wahl anderer Verteilungen hier und auch für die Ziel-VOC könnte die Effizienz oder Performance weiter gesteigert werden, indem z.B. besonders interessante Konzentrationsbereiche mehr ins Training einfließen.

Die in dieser Arbeit untersuchten sechs Gase sind voraussichtlich nicht ausreichend, um Sensoren für Innenraumluft zu charakterisieren. Daher sollte die Komplexität, z. B. durch eine größere Anzahl an Hintergrund-, Stör- und Zielgasen, konsequent gesteigert werden, um sich der Realität weiter anzunähern.

Ein nächster Schritt ist der Aufbau neuer apparativer Lösungen, mit denen sich eine höhere Anzahl von Gasen vermessen lassen.

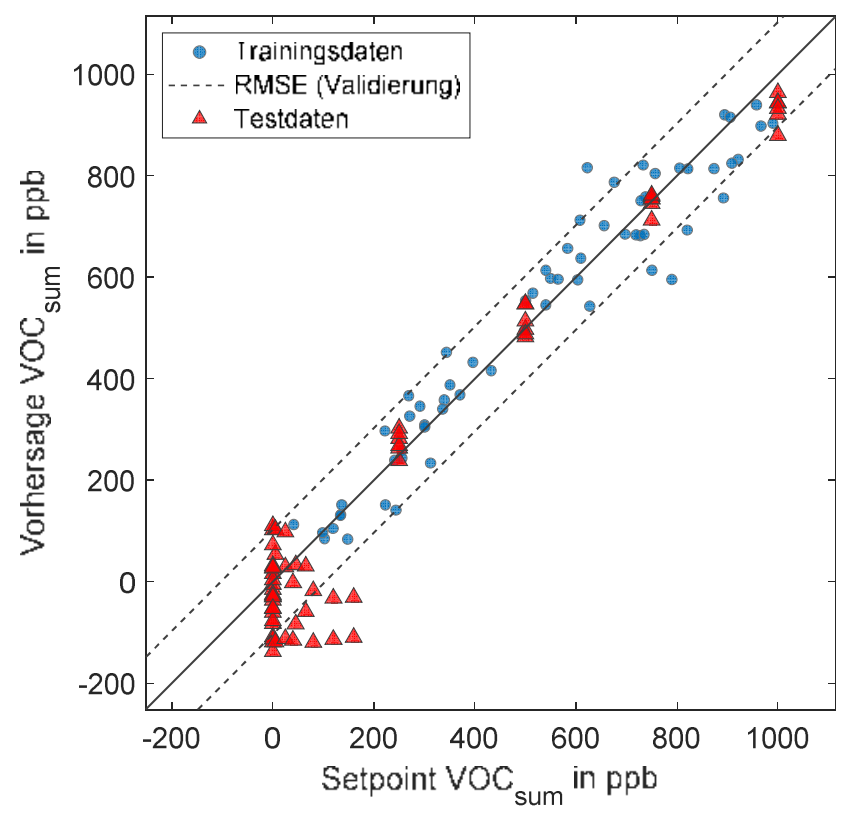

Abb. 6 PLSR-Modell für den AS-MLV-P2 für die Quantifizierung von $\mathrm{VOC}_{\text {sum }}$ in ppb; Datensatz mit 132 Gasgemischen (66 randomisierte und 66 sequenzielle); Training und 6-fache Kreuzvalidierung mit den randomisierten und

Testen mit den sequenziellen Gasgemischen.

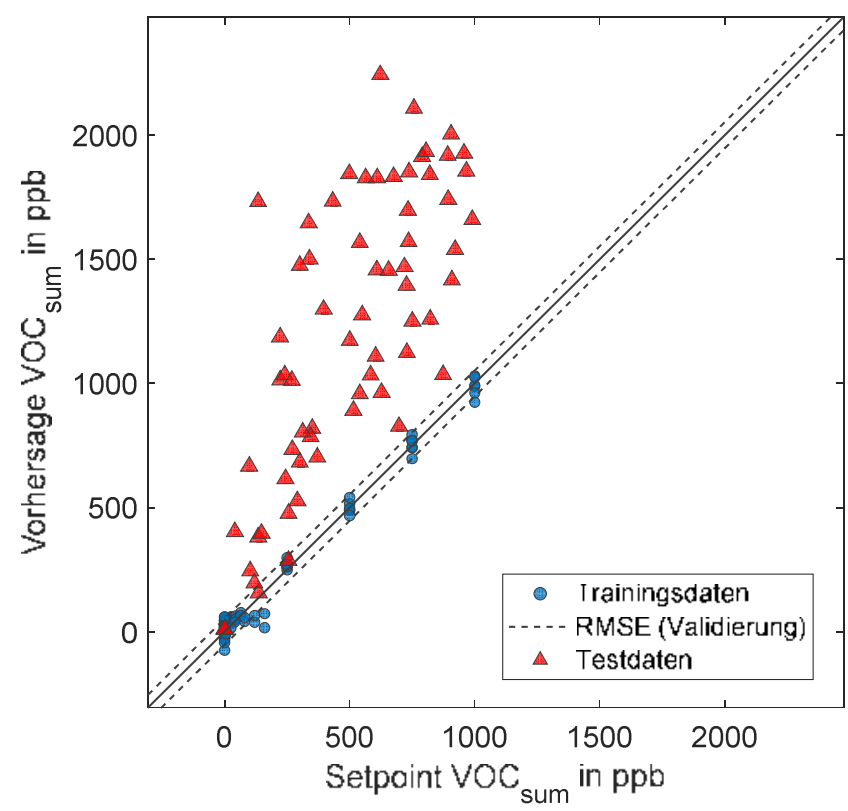

Abb. 7: PLSR-Modell für den AS-MLV-P2 für die Quantifizierung von $\mathrm{VOC}_{\text {sum }}$ in ppb. Datensatz mit 132 Gasgemischen (66 randomisierte und 66 sequenzielle); Training und 6-fache Kreuzvalidierung mit den sequenziellen und Testen mit den randomisierten Gasgemischen. 


\section{Literatur}

[1] FONOLLOSA, J.; RODRÍGUEZ-LUJÁN, I.; HUERTA R.: Chemical gas sensor array dataset, Data in Brief, Vol. 3, 85-89, 2015. doi: 10.1016/j.dib.2015.01.003.

[2] BASTUCK, M.; FRICKE, T.: Temperature-Modulated Gas Sensor Signal, 2018. doi: 10.5281/zenodo.1411209.

[3] WOLFRUM, E. J.; MEGLEN R. M.; PETERSON, D.; SLUITER, J.: Calibration transfer among sensor arrays designed for monitoring volatile organic compounds in indoor air quality, IEEE Sensors Journal, Vol. 6, Nr. 6, 1638-1643, 2006. doi: 10.1109/JSEN.2006.884558

[4] SUNDGREN, H.; WINQUIST F.; LUKKARI, I.; LUNDSTROM, I.: Artificial neural networks and gas sensor arrays: Quantification of individual components in a gas mixture, Measurement Science and Technology, Vol. 2, Nr. 5, 464-469, 1991. doi: 10.1088/09570233/2/5/008

[5] OEHLERT, G. W.; A First Course in Design and Analysis of Experiments, 2000, ISBN: 978-0-7167-3510-6.

[6] BASTUCK., M: Improving the Performance of Gas Sensor Systems with Advanced Data Evaluation, Operation, and Calibration Methods, 2019, Dissertation, Saarland University and Linköping University, doi: 10.3384/diss.diva-159106

[7] GRAMM, A.; SCHÜTZE, A.: High performance solvent vapor identification with a two sensor array using temperature cycling and pattern classification, Sensors \& Actuators B 95 (2003), 58-65, doi: 10.1016/S09254005(03)00404-0

[8] SCHÜTZE, A.; SAUERWALD, T.: Dynamic operation of semiconductor sensors; in: Raivo Jaaniso and Ooi Kiang Tan (eds.): Semiconductor Gas Sensors, Woodhead Publishing Series in Electronic and Optical Materials, 2nd Edition, 2020, doi: 10.1016/B978-0-08102559-8.00012-4

[9] BAUR, T.; SCHULTEALBERT, C.; SCHÜTZE, A.; SAUERWALD, T.: Novel method for the detection of short trace gas pulses with metal oxide semiconductor gas sensors, Journal of Sensors and Sensor Systems, 2018, 7(1), pp. 411-419, doi:10.5194/jsss-7-411-2018

[10] BAUR, T.; SCHULTEALBERT, C.; SCHÜTZE, A.; SAUERWALD, T.: Device for the detection of short trace gas pulses. tm - Technisches Messen, 2017, 85(7-8), 496-503. doi:10.1515/teme-2017-0137

[11] SCHULTEALBERT, C.; BAUR, T.; SCHÜTZE, A.; BÖTTCHER, S.; SAUERWALD, T.: A novel approach towards calibrated measurement of trace gases using metal oxide semiconductor sensors, Sensors \& Actuators: B. Chemical, 2017, 239, 390-396, doi:10.1016/j.snb.2016.08.002

[12] SCHULTEALBERT, C.; BAUR, T.; SCHÜTZE, A.; SAUERWALD, T.: Facile Quantification and Identification Techniques for Reducing Gases over a Wide Concentration Range Using a MOS Sensor in Temperature-Cycled Operation, Sensors, 2018, 18(3), 744, doi: 10.3390/s18030744

[13] HELWIG, N.; SCHÜLER, M.; BUR, C.; SCHÜTZE, A.; SAUERWALD, T.: Gas mixing apparatus for automated gas sensor characterization, Measurement Science and Technology, 2014, 25(5), 055903-055903, doi:10.1088/0957-0233/25/5/055903

[14] LEIDINGER, M.; SCHULTEALBERT, C.; NEU, J.; SCHÜTZE, A.; SAUERWALD, T.: Characterization and calibration of gas sensor systems at ppb level-a versatile test gas generation system, Measurement Science and Technology, 2018, 29(1), doi:10.1088/13616501/aa91da

[15] UMWELTBUNDESAMT: Beurteilung von Innenraumluftkontaminationen mittels Referenz- und Richtwerten, Bundesgesundheitsblatt - Gesundheitsforschung - Gesundheitsschutz, 2017, 50, 990-1005, doi:10.1007/s00103-007-0290-y

[16] HOFMANN, H.; PLIENINGER, P.: Bereitstellung einer Datenbank zum Vorkommen von flüchtigen organischen Verbindungen in der Raumluft, WaBoLu-Hefte 05/08 des Umweltbundesamtes

[17] SCHLEYER, R.; BIEBER, E.; WALLASCH, M.: Das Luftmessnetz des Umweltbundesamtes, 2013.

[18] BASTUCK, M.; BAUR, T.; SCHÜTZE, A.: DAV3E - a MATLAB toolbox for multivariate sensor data evaluation, Journal of Sensors and Sensor Systems, 2018, 7, 489-506, doi: 10.5194/jsss-7-489-2018. 